\title{
A CERTAIN CLASS OF ANALYTIC FUNCTIONS OF COMPLEX ORDER CONNECTED WITH A $q$-ANALOGUE OF INTEGRAL OPERATORS
}

\author{
H. M. SRIVASTAVA AND SHEZA M. EL-DEEB
}

Received 30 October, 2019

\begin{abstract}
In this paper, we introduce a certain class $\mathcal{H}_{m, q}^{\lambda, \alpha}(\zeta, \mathcal{M})$ of normalized analytic functions of complex order connected with a $q$-analogue of integral operators. For this complex-order analytic function class, we determine a sufficient condition in terms of the coefficients, estimates for the coefficients and a maximization theorem concerning the coefficients. Various consequences and applications of our main results are also considered. A brief remark about the demonstrated equivalence of the $q$-calculus and the so-called $(p, q)$-calculus is also presented.
\end{abstract}

2010 Mathematics Subject Classification: 05A30; 30C45; 11B65; 47B38

Keywords: analytic functions, univalent functions, complex-order analytic functions, complexorder univalent functions, integral operators, $q$-numbers and $q$-factorials, $q$-derivative operator, maximization theorem, equivalence of the $q$-calculus and the so-called $(p, q)$-calculus

\section{Introduction, DeFinitions AND PRELIMINARIES}

The theory of $q$-calculus plays an important rôle in many areas of mathematical, physical and engineering sciences. Jackson (see [8] and [9]) was the first to have some applications of the $q$-calculus and introduced the $q$-analogue of the classical derivative and integral operators (see also [1]). Let $\mathcal{A}$ denote the class of functions $f(z)$ of the following normalized form:

$$
f(z)=z+\sum_{k=2}^{\infty} a_{k} z^{k} \quad(z \in \mathbb{U})
$$

which are analytic in the open unit disk $\mathbb{U}$ given by

$$
\mathbb{U}=\{z: z \in \mathbb{C} \text { and }|z|<1\}) .
$$

We also let $\mathcal{S}$ denote the subclass of $\mathcal{A}$ consisting of normalized analytic functions which are univalent in $\mathbb{U}$. 
For a function $f(z)$ given by (1.1) and the $g(z)$ given by

$$
g(z)=z+\sum_{k=2}^{\infty} b_{k} z^{k} \quad(z \in \mathbb{U})
$$

the Hadamard product (or convolution) of $f(z)$ and $g(z)$ is defined here by

$$
(f * g)(z):=z+\sum_{k=2}^{\infty} a_{k} b_{k} z^{k}=:(g * f)(z) .
$$

We use $\Omega$ to denote the class of Schwarz functions $w(z)$, which are analytic in $U$ and satisfy the conditions

$$
w(0)=0 \quad \text { and } \quad|w(z)|<1 \quad(z \in \mathbb{U}) .
$$

We now define the integral operator $\mathcal{K}_{m, n}^{\alpha}: \mathcal{A} \rightarrow \mathcal{A}$ for $\alpha>0$ and $m \geqq 0$ as follows:

$$
\mathcal{K}_{m}^{0} f(z)=f(z)
$$

and

$$
\mathcal{K}_{m}^{\alpha} f(z)=\frac{(m+1)^{\alpha}}{\Gamma(\alpha) z^{m}} \int_{0}^{z} t^{m-1}\left(\log \frac{z}{t}\right)^{\alpha-1} f(t) d t .
$$

For $f \in \mathcal{A}$, it can be easily verified that

$$
\mathcal{K}_{m n}^{\alpha} f(z)=z+\sum_{k=2}^{\infty}\left(\frac{m+1}{m+k}\right)^{\alpha} a_{k} z^{k}
$$

Next, for $0<q<1$, the $q$-derivative of the function $\mathcal{K}_{m}^{\alpha} f(z) \in \mathcal{A}$ is defined by

$$
D_{q}\left\{\mathcal{K}_{m}^{\alpha} f(z)\right\}=\frac{\mathcal{K}_{m}^{\alpha} f(z)-\mathcal{K}_{m}^{\alpha} f(q z)}{z(1-q)} \quad(z \neq 0),
$$

so that

$$
D_{q}\left\{z+\sum_{k=2}^{\infty}\left(\frac{m+1}{m+k}\right)^{\alpha} a_{k} z^{k}\right\}=1+\sum_{k=2}^{\infty}\left(\frac{m+1}{m+k}\right)^{\alpha}[k]_{q} a_{k} z^{k-1},
$$

where

$$
[k]_{q}:=\frac{1-q^{k}}{1-q}=1+\sum_{j=1}^{k-1} q^{j} \quad \text { and } \quad[0]_{q}=0 .
$$

Remark 1. The first usage of the above-defined $q$-derivative operator $D_{q}$ in Geometric Function Theory was made in 1990 by Ismail et al. [7] (see also [1]). Moreover, a firm footing of the usage of the $q$-calculus in the context of Geometric Function Theory was actually provided and the basic (or $q$-) hypergeometric functions were first used in Geometric Function Theory in a 1989 book-chapter by Srivastava (see, for details, [21]). Several recent developments on various applications of the the $q$ derivative operator $D_{q}$ in Geometric Function Theory can be found in (for example) $[2,13,14,16,18,22-24,26]$. 
It is easily seen from (1.6) that

$$
z D_{q}\left\{\mathcal{K}_{m}^{\alpha} f(z)\right\}=z+\sum_{k=2}^{\infty}\left(\frac{m+1}{m+k}\right)^{\alpha}[k]_{q} a_{k} z^{k}
$$

For any non-negative integer $n$, the $q$-factorial $[n]_{q}$ ! is given by

$$
[n]_{q} != \begin{cases}1 & (k=0) \\ {[1]_{q}[2]_{q}[3]_{q} \cdots[n]_{q}} & (n \in \mathbb{N}),\end{cases}
$$

where $\mathbb{N}$ denotes the set positive integers. Also the $q$-Pochhammer symbol $[\lambda]_{q, n}$ $(v \in \mathbb{C})$ is defined by

$$
[v]_{q, n}= \begin{cases}1 & (n=0) \\ {[v]_{q}[v+1]_{q} \cdots[v+n-1]_{q}} & (n \in \mathbb{N}) .\end{cases}
$$

For $\lambda>-1$, we define the operator $\mathcal{N}_{m, q}^{\lambda, \alpha}$ by

$$
\mathcal{N}_{m, q}^{\lambda, \alpha} f(z) * \mathcal{M}_{q, \lambda+1}(z)=z D_{q}\left\{\mathcal{K}_{m}^{\alpha} f(z)\right\}
$$

where the function $\mathcal{M}_{q, \lambda+1}(z)$ is given by

$$
\mathcal{M}_{q, \lambda+1}(z)=z+\sum_{k=2}^{\infty} \frac{[\lambda+1]_{q, k-1}}{[k-1]_{q} !} z^{k} .
$$

We thus obtain

$$
\begin{aligned}
\mathcal{N}_{m, q}^{\lambda, \alpha} f(z) & =z+\sum_{k=2}^{\infty}\left(\frac{m+1}{m+k}\right)^{\alpha} \frac{[k]_{q}[k-1]_{q} !}{[\lambda+1]_{q, k-1}} a_{k} z^{k} \\
& =z+\sum_{k=2}^{\infty} \frac{[k]_{q} !}{[\lambda+1]_{q, k-1}}\left(\frac{m+1}{m+k}\right)^{\alpha} a_{k} z^{k} \\
(\alpha & >0 ; \lambda>-1 ; m \geqq 0 ; 0<q<1) .
\end{aligned}
$$

We can easily verify from (1.11) that

$$
[\lambda+1]_{q} \mathcal{N}_{m, q}^{\lambda, \alpha} f(z)=[\lambda]_{q} \mathcal{N}_{m, q}^{\lambda+1, \alpha} f(z)+q^{\lambda} z D_{q}\left\{\mathcal{N}_{m, q}^{\lambda+1, \alpha} f(z)\right\}
$$

We also note that

$$
\lim _{q \rightarrow 1-} \mathcal{N}_{m, q}^{\lambda, \alpha} f(z)=I_{m}^{\lambda, \alpha} f(z)=z+\sum_{k=2}^{\infty} \frac{k !}{(\lambda+1)_{k-1}}\left(\frac{m+1}{m+k}\right)^{\alpha} a_{k} z^{k}
$$

In the special case when $\alpha=0$, we have

$$
\mathcal{N}_{m, q}^{\lambda, 0} f(z)=: \mathfrak{J}_{q}^{\lambda} f(z) .
$$

The operator in $\mathfrak{J}_{q}^{\lambda} f(z)$ was studied by Arif et al. [5]. 
Definition 1. We say that a function $f(z)$ belonging to $\mathcal{A}$ is in the normalized complex-order analytic function class

$$
\mathcal{H}_{m, q}^{\lambda, \alpha}(\zeta, \mathcal{M}) \quad\left(\zeta \in \mathbb{C}^{*}:=\mathbb{C} \backslash\{0\} ; \mathcal{M}>\frac{1}{2}\right)
$$

if and only if

$$
\begin{gathered}
\left|1-\frac{1}{\zeta}+\frac{z\left(\mathcal{N}_{m, q}^{\lambda, \alpha} f(z)\right)^{\prime}}{\zeta \mathcal{N}_{m, q}^{\lambda, \alpha} f(z)}-\mathcal{M}\right|_{(\alpha>0 ; \lambda>-1 ; m \geqq 0 ; 0<q<1 ; z \in \mathbb{U}) .}<\mathcal{M}
\end{gathered}
$$

By letting $q \rightarrow 1-$, it follows from the work of Kulshrestha [11] that

$$
g(z) \in \mathcal{H}_{m, q}^{1,0}(1, \mathcal{M})=F(1, \mathcal{M})
$$

if and only if

$$
\frac{z g^{\prime}(z)}{g(z)}=\frac{1+w(z)}{1-m w(z)} \quad\left(m=1-\frac{1}{\mathcal{M}} ; \mathcal{M}>\frac{1}{2} ; w(z) \in \Omega\right)
$$

for $z \in \mathbb{U}$.

It can easily be shown that $f(z) \in \mathcal{H}_{m, q}^{\lambda, \alpha}(\zeta, \mathcal{M})$ if and only if there exists a function

$$
g(z) \in \lim _{q \rightarrow 1-} \mathcal{H}_{m, q}^{1,0}(1, \mathcal{M})=F(1, \mathcal{M})
$$

such that

$$
\mathcal{N}_{m, q}^{\lambda, \alpha} f(z)=z\left[\frac{g(z)}{z}\right]^{\zeta}
$$

Thus, from (1.15) and (1.16), it follows that $f(z) \in \mathcal{H}_{m, q}^{\lambda, \alpha}(\zeta, \mathcal{M})$ if and only if

$$
\begin{gathered}
\frac{z\left(\mathcal{N}_{b, q}^{\lambda, \alpha} f(z)\right)^{\prime}}{\mathcal{N}_{m, q}^{\lambda, \alpha} f(z)}=\frac{1+[\zeta(1+m)-m] w(z)}{1-m w(z)} \\
\left(m=1-\frac{1}{\mathcal{M}} ; \mathcal{M}>\frac{1}{2} ; w(z) \in \Omega\right)
\end{gathered}
$$

for $z \in \mathbb{U}$.

By giving specific values to the parameters $\lambda, \alpha$ and $\zeta$, we obtain the following interesting subclasses:

(i) $\lim _{q \rightarrow 1-} \mathcal{H}_{m, q}^{1,0}(\zeta, \mathcal{M})=F(\zeta, \mathcal{M}) \quad$ (see Nasr and Aouf [17]);

(ii) $\lim _{q \rightarrow 1-} \mathcal{H}_{m, q}^{1,0}(1, \mathcal{M})=F(1, \mathcal{M})$ (see Singh and Singh [19]);

(iii) $\lim _{q \rightarrow 1-} \mathcal{H}_{m, q}^{1,0}\left(\cos \lambda e^{-i \lambda}, \mathcal{M}\right)=F_{\lambda, \mathscr{M}} \quad\left(|\lambda|<\frac{\pi}{2}\right) \quad$ (see Kulshrestha [11]); 
(iv) $\lim _{q \rightarrow 1-} \mathcal{H}_{m, q}^{1,0}\left((1-\alpha) \cos \lambda e^{-i \lambda}, \infty\right)=S^{\lambda}(\alpha) \quad\left(|\lambda|<\frac{\pi}{2} ; 0 \leqq \alpha<1\right)$ (see Libera [12]; see also Chichra [6] and Sižuk [20]);

(v) $\lim _{q \rightarrow 1-} \mathcal{H}_{m, q}^{1,0}\left((1-\alpha) \cos \lambda e^{-i \lambda}, \mathcal{M}\right)=F_{\mathcal{M}}(\lambda, \alpha) \quad\left(|\lambda|<\frac{\pi}{2} ; 0 \leqq \alpha<1\right)$ (see Aouf [3] and Aouf [4]).

We also have the following presumably new function classes:

(i) $\lim _{q \rightarrow 1-} \mathcal{H}_{m, q}^{\lambda, \alpha}(\zeta, \mathcal{M})=: \mathcal{S}_{m}^{\lambda, \alpha}(\zeta, \mathcal{M})$, where

$$
\begin{array}{r}
\mathcal{S}_{m}^{\lambda, \alpha}(\zeta, \mathcal{M}):=\left\{f: f(z) \in \mathcal{A} \text { and }\left|1-\frac{1}{\zeta}+\frac{z\left(I_{m}^{\lambda, \alpha} f(z)\right)^{\prime}}{\zeta I_{m}^{\lambda, \alpha} f(z)}-\mathcal{M}\right|<\mathcal{M}\right. \\
\left.\quad\left(M>\frac{1}{2} ; \zeta \in \mathbb{C}^{*} ; \alpha>0 ; \lambda>-1 ; m \geqq 0 ; z \in \mathbb{U}\right)\right\} ;
\end{array}
$$

(ii) $\mathcal{H}_{m, q}^{\lambda, 0}(\zeta, \mathcal{M})=: \mathcal{F}_{q}^{\lambda}(\zeta, \mathcal{M})$, where

$$
\begin{aligned}
\mathcal{F}_{q}^{\lambda}(\zeta, \mathcal{M}):= & \left\{f: f(z) \in \mathcal{A} \text { and }\left|1-\frac{1}{\zeta}+\frac{z\left(\mathfrak{J}_{q}^{\lambda} f(z)\right)^{\prime}}{\zeta \mathfrak{J}_{q}^{\lambda} f(z)}-\mathcal{M}\right|<\mathcal{M}\right. \\
& \left.\left(\mathcal{M}>\frac{1}{2} ; \zeta \in \mathbb{C}^{*} ; \lambda>-1 ; m \geqq 0 ; 0<q<1 ; z \in \mathbb{U}\right)\right\} .
\end{aligned}
$$

From the above definitions of the function classes $F(\zeta, \mathcal{M})$ and $\mathcal{H}_{m, q}^{\lambda, \alpha}(\zeta, \mathcal{M})$, we note that

$$
f(z) \in \mathcal{H}_{m, q}^{\lambda, \alpha}(\zeta, \mathcal{M}) \quad \Longleftrightarrow \quad \mathcal{N}_{m, q}^{\lambda, \alpha} f(z) \in F(\zeta, \mathcal{M})
$$

The purpose of the present paper is to determine a sufficient condition in terms of the coefficients for functions belonging to the normalized complex-order analytic function class $\mathcal{H}_{m, q}^{\lambda, \alpha}(\zeta, \mathcal{M})$, estimates for the coefficients and a maximization theorem involving $\left|a_{3}-\mu a_{2}^{2}\right|$ for the class $\mathcal{H}_{m, q}^{\lambda, \alpha}(\zeta, \mathcal{M})$ for complex values of the parameter $\mu$.

\section{Sufficient Condition for a FunCtion to BE In the Class $\mathcal{H}_{m, q}^{\lambda, \alpha}(\zeta, \mathcal{M})$}

Unless otherwise mentioned, we assume throughout this paper that

$$
\begin{gathered}
\alpha>0, \lambda>-1, m \geqq 0,0<q<1, \zeta \in \mathbb{C}^{*}, \\
m=1-\frac{1}{\mathcal{M}}, \mathcal{M}>\frac{1}{2} \quad \text { and } \quad z \in \mathbb{U} .
\end{gathered}
$$


Theorem 1. Let the function $f(z)$ be defined by (1.1). Also let the following inequality holds true:

$$
\sum_{k=2}^{\infty}\{(k-1)+|\zeta(1+m)+m(k-1)|\}\left|a_{k}\right| \frac{[k]_{q} !}{[\lambda+1]_{q, k-1}}\left(\frac{m+1}{m+k}\right)^{\alpha}|\zeta(1+m)| .
$$

Then $f(z)$ belongs to the class normalized complex-order analytic function class $\mathcal{H}_{m, q}^{\lambda, \alpha}(\zeta, \mathcal{M})$.

Proof. Suppose that the inequality (2.1) holds true. Then we find for $z \in \mathbb{U}$ that

$$
\begin{aligned}
\mid z & \left(\mathcal{N}_{m, q}^{\lambda, \alpha} f(z)\right)^{\prime}-\mathcal{N}_{m, q}^{\lambda, \alpha} f(z)|-| \zeta(1+m) \mathcal{N}_{m, q}^{\lambda, \alpha} f(z)+m\left\{z\left(\mathcal{N}_{m, q}^{\lambda, \alpha} f(z)\right)^{\prime}-\mathcal{N}_{m, q}^{\lambda, \alpha} f(z)\right\} \mid \\
= & \left|\sum_{k=2}^{\infty}(k-1) \frac{[k]_{q} !}{[\lambda+1]_{q, k-1}}\left(\frac{m+1}{m+k}\right)^{\alpha} a_{k} z^{k}\right| \\
& -\mid \zeta(1+m)\left\{z+\sum_{k=2}^{\infty} \frac{[k]_{q} !}{[\lambda+1]_{q, k-1}}\left(\frac{m+1}{m+k}\right)^{\alpha} a_{k} z^{k}\right\} \\
& +m\left\{\sum_{k=2}^{\infty}(k-1) \frac{[k]_{q} !}{[\lambda+1]_{q, k-1}}\left(\frac{m+1}{m+k}\right)^{\alpha} a_{k} z^{k}\right\} \mid \\
= & \left|\sum_{k=2}^{\infty}(k-1) \frac{[k]_{q} !}{[\lambda+1]_{q, k-1}}\left(\frac{m+1}{m+k}\right)^{\alpha} a_{k} z^{k}\right| \\
& -\left|\zeta(1+m) z+\sum_{k=2}^{\infty}\{\zeta(1+m)+m(k-1)\} \cdot \frac{[k]_{q} !}{[\lambda+1]_{q, k-1}}\left(\frac{m+1}{m+k}\right)^{\alpha} a_{k} z^{k}\right| \\
\leqq & \sum_{k=2}^{\infty}(k-1) \frac{[k]_{q} !}{[\lambda+1]_{q, k-1}}\left(\frac{m+1}{m+k}\right)^{\alpha}\left|a_{k}\right| r^{k} \\
& -\left\{|\zeta(1+m)| r-\sum_{k=2}^{\infty}\{|\zeta(1+m)|+m(k-1)\} \cdot \frac{[k]_{q} !}{[\lambda+1]_{q, k-1}}\left(\frac{m+1}{m+k}\right)^{\alpha}\left|a_{k}\right| r^{k}\right\} \\
= & \sum_{k=2}^{\infty}\{(k-1)+|\zeta(1+m)|+m(k-1)\} \cdot \frac{[k]_{q} !}{[\lambda+1]_{q, k-1}}\left(\frac{m+1}{m+k}\right)^{\alpha}\left|a_{k}\right| r^{k}-|\zeta(1+m)| r .
\end{aligned}
$$

Letting $r \rightarrow 1-$ in the above equation, we get

$$
\begin{aligned}
& \left|z\left(\mathcal{N}_{m, q}^{\lambda, \alpha} f(z)\right)^{\prime}-\mathcal{N}_{m, q}^{\lambda, \alpha} f(z)\right|-\left|\zeta(1+m) \mathcal{N}_{m, q}^{\lambda, \alpha} f(z)+m\left\{z\left(\mathcal{N}_{m, q}^{\lambda, \alpha} f(z)\right)^{\prime}-\mathcal{N}_{m, q}^{\lambda, \alpha} f(z)\right\}\right| \\
& \leqq \sum_{k=2}^{\infty}\{(k-1)+|\zeta(1+m)|+m(k-1)\}\left|a_{k}\right| \cdot \frac{[k]_{q} !}{[\lambda+1]_{q, k-1}}\left(\frac{m+1}{m+k}\right)^{\alpha}-|\zeta(1+m)| \leqq 0,
\end{aligned}
$$


where we have made use of the assertion (2.1) of Theorem 1. Consequently, we obtain

$$
\left|\frac{\frac{z\left(\mathcal{N}_{m, q}^{\lambda, \alpha} f(z)\right)^{\prime}}{\mathcal{N}_{m, q}^{\lambda, \alpha} f(z)}-1}{\zeta(1+m)+m\left(\frac{z\left(\mathcal{N}_{m, q}^{\lambda, \alpha} f(z)\right)^{\prime}}{\mathcal{N}_{m, q}^{\lambda, \alpha} f(z)}-1\right)}\right|<1 \quad(z \in \mathbb{U}) .
$$

If we now set

$$
w(z)=\frac{\frac{z\left(\mathcal{N}_{m, q}^{\lambda, \alpha} f(z)\right)^{\prime}}{\mathcal{N}_{m, q}^{\lambda, \alpha} f(z)}-1}{\zeta(1+m)+m\left(\frac{z\left(\mathcal{N}_{m, q, \alpha}^{\lambda, \alpha} f(z)\right)^{\prime}}{\mathcal{N}_{m, q}^{\lambda, \alpha} f(z)}-1\right)},
$$

then $w(0)=0, w(z)$ is analytic in the open unit disk $\mathbb{U}$ and

$$
|w(z)|<1 \quad(z \in \mathbb{U}) .
$$

Hence we have

$$
\frac{z\left(\mathcal{N}_{m, q}^{\lambda, \alpha} f(z)\right)^{\prime}}{\mathcal{N}_{m, q}^{\lambda, \alpha} f(z)}=\frac{1+[\zeta(1+m)-m] w(z)}{1-m w(z)},
$$

which shows that the function $f(z)$ belongs to the class $\mathcal{H}_{m, q}^{\lambda, \alpha}(\zeta, \mathcal{M})$.

In the limit when $q \rightarrow 1-$ in Theorem 1, we obtain the following corollary.

Corollary 1. Let the function $f(z)$ be defined by (1.1). Also let the following inequality holds true:

$$
\sum_{k=2}^{\infty}\{(k-1)+|\zeta(1+m)+m(k-1)|\}\left|a_{k}\right| \cdot \frac{k !}{(\lambda+1)_{k-1}}\left(\frac{m+1}{m+k}\right)^{\alpha} \leqq|\zeta(1+m)| .
$$

Then the function $f(z)$ belongs to the class $\mathcal{S}_{m}^{\lambda, \alpha}(\zeta, \mathcal{M})$.

If we set $\alpha=0$ in Theorem 1, we obtain the following corollary.

Corollary 2. Let the function $f(z)$ be defined by (1.1). Also let the following inequality holds true:

$$
\sum_{k=2}^{\infty}\{(k-1)+|\zeta(1+m)+m(k-1)|\}\left|a_{k}\right| \frac{[k]_{q} !}{[\lambda+1]_{q, k-1}} \leqq|\zeta(1+m)| .
$$

Then the function $f(z)$ belongs to the class $\mathcal{F}_{q}^{\lambda}(\zeta, \mathcal{M})$. 


\section{Coefficient Estimates}

In this section, we first state and prove the following result.

Theorem 2. Let the function $f(z)$ given by (1.1) be in the normalized complexorder analytic function class $\mathcal{H}_{m, q}^{\lambda, \alpha}(\zeta, \mathcal{M})$.

(a) If

$$
2 m(k-1) \Re(\zeta)>(k-1)^{2}(1-m)-|\zeta|^{2}(1+m),
$$

let

$$
\mathcal{G}=\left[\frac{2 m(k-1) \Re(\zeta)}{(k-1)^{2}(1-m)-|\zeta|^{2}(1+m)}\right] \quad(k=2,3,4, \cdots, j-1),
$$

where $\mathcal{N}=[\mathcal{G}]$ (the Gaussian symbol) and $[\mathcal{G}]$ is the greatest integer not greater than $\mathcal{G}$. Then

$$
\begin{gathered}
\left|a_{j}\right| \leqq \frac{[\lambda+1]_{q, j-1}}{[j]_{q} !\left(\frac{m+1}{m+j}\right)^{\alpha}(j-1) !} \prod_{k=2}^{j}|\zeta(1+m)+m(k-2)| \\
(j=2,3,4, \cdots, \mathcal{N}+2)
\end{gathered}
$$

and

$$
\begin{gathered}
\left|a_{j}\right| \leqq \frac{[\lambda+1]_{q, j-1}}{[j]_{q} !(j-1)\left(\frac{m+1}{m+j}\right)^{\alpha}(\mathcal{N}+1) !} \cdot \prod_{k=2}^{\mathcal{N}+3}|\zeta(1+m)+m(k-2)| . \\
(j>\mathcal{N}+2)
\end{gathered}
$$

(b) If

$$
2 m(k-1) \Re(\zeta) \leqq(k-1)^{2}(1-m)-|\zeta|^{2}(1+m),
$$

then

$$
\left|a_{j}\right| \leqq \frac{[\lambda+1]_{q, j-1}(1+m)|\zeta|}{\left(\frac{m+1}{m+j}\right)^{\alpha}[j]_{q} !(j-1)} \quad(j \geqq 2) .
$$

The inequalities (3.1) and (3.3) are sharp.

Proof. Let us assume that $f(z) \in \mathcal{H}_{m, q}^{\lambda, \alpha}(\zeta, \mathcal{M})$. Then we find from (1.16) that

$$
\begin{aligned}
& \sum_{k=2}^{\infty}(k-1) \frac{[k]_{q} !}{[\lambda+1]_{q, k-1}}\left(\frac{m+1}{m+k}\right)^{\alpha} a_{k} z^{k} \\
& =\left\{\zeta(1+m) z+\sum_{k=2}^{\infty}\{\zeta(1+m)+m(k-1)\} \cdot \frac{[k]_{q} !}{[\lambda+1]_{q, k-1}}\left(\frac{m+1}{m+k}\right)^{\alpha} a_{k} z^{k}\right\} w(z),
\end{aligned}
$$


which is equivalent to

$$
\begin{aligned}
& \sum_{k=2}^{j}(k-1) \frac{[k]_{q} !}{[\lambda+1]_{q, k-1}}\left(\frac{m+1}{m+k}\right)^{\alpha} a_{k} z^{k}+\sum_{k=j+1}^{\infty} c_{k} z^{k} \\
& =\left\{\zeta(1+m) z+\sum_{k=2}^{j-1}\{\zeta(1+m)+m(k-1)\} \cdot \frac{[k]_{q} !}{[\lambda+1]_{q, k-1}}\left(\frac{m+1}{m+k}\right)^{\alpha} a_{k} z^{k}\right\} w(z),
\end{aligned}
$$

where the coefficients $c_{j}$ are some complex numbers and the series $\sum_{k=j+1}^{\infty} c_{k} z^{k}$ converges when $z \in \mathbb{U}$. Then, since

$$
|w(z)|<1 \quad(z \in \mathbb{U}),
$$

we have

$$
\begin{aligned}
& \left|\sum_{k=2}^{j}(k-1) \frac{[k]_{q} !}{[\lambda+1]_{q, k-1}}\left(\frac{m+1}{m+k}\right)^{\alpha} a_{k} z^{k}+\sum_{k=j+1}^{\infty} c_{k} z^{k}\right| \\
& \leqq \zeta(1+m) z+\sum_{k=2}^{j-1}\{\zeta(1+m)+m(k-1)\} \cdot \frac{[k]_{q} !}{[\lambda+1]_{q, k-1}}\left(\frac{m+1}{m+k}\right)^{\alpha} a_{k} z^{k} \mid .
\end{aligned}
$$

Squaring both sides of (3.5), we get

$$
\begin{aligned}
& \sum_{k=2}^{j}(k-1)^{2}\left(\frac{[k]_{q} !}{[\lambda+1]_{q, k-1}}\right)^{2}\left(\frac{m+1}{m+k}\right)^{2 \alpha}\left|a_{k}\right|^{2} r^{2 k}+\sum_{k=j+1}^{\infty}\left|c_{k}\right|^{2} r^{2 k} \\
& \leqq \\
& \qquad(1+m)^{2}|\zeta|^{2} r^{2}+\sum_{k=2}^{j-1}|\zeta(1+m)+m(k-1)|^{2}\left(\frac{[k]_{q} !}{[\lambda+1]_{q, k-1}}\right)^{2} \\
& \left.\quad \cdot\left(\frac{m+1}{m+k}\right)^{2 \alpha}\left|a_{k}\right|^{2} r^{2 k}\right\} .
\end{aligned}
$$

We now let $r \rightarrow 1-$. Then, on some simplification, we obtain

$$
\begin{aligned}
& (j-1)^{2}\left|a_{j}\right|^{2}\left(\frac{[j]_{q} !}{[\lambda+1]_{q, j-1}}\right)^{2}\left(\frac{m+1}{m+j}\right)^{2 \alpha} \\
& \leqq(1+m)^{2}|\zeta|^{2}+\sum_{k=2}^{j-1}\left\{|\zeta(1+m)+m(k-1)|^{2}-(k-1)^{2}\right\} \\
& \quad \cdot\left|a_{k}\right|^{2}\left(\frac{[k]_{q} !}{[\lambda+1]_{q, k-1}}\right)^{2}\left(\frac{m+1}{m+k}\right)^{2 \alpha} .
\end{aligned}
$$

The following two cases arise:

(a) Let

$$
2 m(k-1) \Re(\zeta)>(k-1)^{2}(1-m)-|\zeta|^{2}(1+m) .
$$


Suppose also that $j \leqq \mathcal{N}+2$. Then, for $j=2$, the equation (3.6) gives

$$
\left|a_{2}\right| \leqq \frac{(1+m)[\lambda+1]_{q, 1}|\zeta|}{[2]_{q} !\left(\frac{m+1}{m+2}\right)^{\alpha}}
$$

which yields (3.1) for $j=2$. We establish the assertion (3.1) by appealing to the principle of mathematical induction. Suppose (3.1) is valid for $k=2,3,4, \cdots, j-1$. Then, clearly, it follows from (3.6) that

$$
\begin{aligned}
&(j-1)^{2}\left|a_{j}\right|^{2}\left(\frac{[j]_{q} !}{[\lambda+1]_{q, j-1}}\right)^{2}\left(\frac{m+1}{m+j}\right)^{2 \alpha} \\
& \leqq(1+m)^{2}|\zeta|^{2}+\sum_{k=2}^{j-1}\left(\frac{[k]_{q} !}{[\lambda+1]_{q, k-1}}\right)^{2}\left(\frac{m+1}{m+k}\right)^{2 \alpha} \\
& \cdot\left\{|\zeta(1+m)+m(k-1)|^{2}-(k-1)^{2}\right\} \\
& \cdot \frac{\left([\lambda+1]_{q, k-1}\right)^{2}}{\left([k]_{q} !\right)^{2}\left(\frac{m+1}{m+k}\right)^{2 \alpha}((k-1) !)^{2}} \prod_{p=2}^{k}|\zeta(1+m)+m(p-2)|^{2} \\
&= \frac{1}{((j-2) !)^{2}} \prod_{k=2}^{j}|\zeta(1+m)+m(k-2)|^{2} .
\end{aligned}
$$

We thus find that

$$
\left|a_{j}\right| \leqq \frac{[\lambda+1]_{q, j-1}}{[j]_{q} !\left(\frac{m+1}{m+j}\right)^{\alpha}(j-1) !} \prod_{k=2}^{j}|\zeta(1+m)+m(k-2)|,
$$

which completes the proof of the assertion (3.1) of Theorem 2 .

We next suppose that $j>\mathcal{N}+2$. Then (3.6) gives

$$
\begin{aligned}
&(j-1)^{2}\left|a_{j}\right|^{2}\left(\frac{[j, q] !}{[\lambda+1]_{q, j-1}}\right)^{2}\left(\frac{m+1}{m+j}\right)^{2 \alpha} \\
& \leqq(1+m)^{2}|\zeta|^{2}+\sum_{k=2}^{\mathcal{K}+2}\left(\frac{[k]_{q} !}{[\lambda+1]_{q, k-1}}\right)^{2}\left(\frac{m+1}{m+k}\right)^{2 \alpha} \\
& \cdot\left\{|\zeta(1+m)+m(k-1)|^{2}-(k-1)^{2}\right\}\left|a_{k}\right|^{2} \\
&+\sum_{k=\mathcal{N}+3}^{j-1}\left(\frac{[k]_{q} !}{[\lambda+1]_{q, k-1}}\right)^{2}\left(\frac{m+1}{m+k}\right)^{2 \alpha}
\end{aligned}
$$




$$
\begin{aligned}
& \cdot\left\{|\zeta(1+m)+m(k-1)|^{2}-(k-1)^{2}\right\}\left|a_{k}\right|^{2} \\
\leqq & (1+m)^{2}|\zeta|^{2}+\sum_{k=2}^{\mathcal{K}+2}\left(\frac{[k]_{q} !}{[\lambda+1]_{q, k-1}}\right)^{2}\left(\frac{m+1}{m+k}\right)^{2 \alpha} \\
& \cdot\left\{|\zeta(1+m)+m(k-1)|^{2}-(k-1)^{2}\right\}\left|a_{k}\right|^{2} .
\end{aligned}
$$

Upon substituting the above-derived upper estimates for $a_{2}, a_{3}, \cdots, a_{\mathfrak{N}+2}$ if we simplify the resulting equations, we obtain the assertion (3.2) of Theorem 2.

(b) If we let

$$
2 m(k-1) \Re(\zeta) \leqq(k-1)^{2}(1-m)-|\zeta|^{2}(1+m),
$$

then it follows from (3.6) that

$$
\left(\frac{[j]_{q} !}{[\lambda+1,]_{q, j-1}}\right)^{2}\left(\frac{m+1}{m+j}\right)^{2 \alpha}(j-1)^{2}\left|a_{j}\right|^{2} \leqq(1+m)^{2}|\zeta|^{2} \quad(j \geqq 2),
$$

which proves the assertion (3.3) of Theorem 2.

Taking $q \rightarrow 1-$ in Theorem 2, we obtain the following corollary.

Corollary 3. Let the function $f(z)$ defined by (1.1) be in the class $\mathcal{S}_{m}^{\lambda, \alpha}(\zeta, \mathcal{M})$.

(a) If

$$
2 m(k-1) \Re(\zeta)>(k-1)^{2}(1-m)-|\zeta|^{2}(1+m),
$$

let

$$
\mathcal{G}=\left[\frac{2 m(k-1) \Re(\zeta)}{(k-1)^{2}(1-m)-|\zeta|^{2}(1+m)}\right] \quad(k=2,3,4, \cdots, j-1),
$$

where $\mathcal{N}=[\mathcal{G}]$ (the Gaussian symbol) and $[\mathcal{G}]$ is the greatest integer not greater than $\mathcal{G}$. Then

$$
\begin{gathered}
\left|a_{j}\right| \leqq \frac{(\lambda+1)_{k-1}}{\left(\frac{m+1}{m+j}\right)^{\alpha} k !(j-1) !} \prod_{k=2}^{j}|\zeta(1+m)+m(k-2)| \\
(j=2,3,4, \cdots, \mathcal{N}+2)
\end{gathered}
$$

and

$$
\begin{gathered}
\left|a_{j}\right| \leqq \frac{(\lambda+1)_{k-1}}{(j-1) k !\left(\frac{m+1}{m+j}\right)^{\alpha}(\mathcal{N}+1) !} \prod_{k=2}^{\mathcal{N}+3}|\zeta(1+m)+m(k-2)| \\
(j>\mathcal{N}+2) .
\end{gathered}
$$


(b) If

$$
2 m(k-1) \Re(\zeta) \leqq(k-1)^{2}(1-m)-|\zeta|^{2}(1+m),
$$

then

$$
\left|a_{j}\right| \leqq \frac{(\lambda+1)_{k-1}(1+m)|\zeta|}{\left(\frac{m+1}{m+j}\right)^{\alpha} k !(j-1)} \quad(j \geqq 2) .
$$

The inequalities (3.7) and (3.9) are sharp.

If we set $\alpha=0$ in Theorem 2, then we obtain the following corollary.

Corollary 4. Let the function $f(z)$ be defined by $(1.1)$ be in the class $\mathcal{F}_{q}^{\lambda}(\zeta, \mathcal{M})$. (a) If

$$
2 m(k-1) \Re(\zeta)>(k-1)^{2}(1-m)-|\zeta|^{2}(1+m),
$$

let

$$
\mathcal{G}=\left[\frac{2 m(k-1) \mathfrak{R}(\zeta)}{(k-1)^{2}(1-m)-|\zeta|^{2}(1+m)}\right] \quad(k=2,3,4, \cdots, j-1),
$$

where $\mathcal{N}=[\mathcal{G}]$ (the Gaussian symbol) and $[\mathcal{G}]$ is the greatest integer not greater than $\mathcal{G}$. Then

$$
\begin{gathered}
\left|a_{j}\right| \leqq \frac{[\lambda+1]_{q, j-1}}{[j]_{q} !(j-1) !} \prod_{k=2}^{j}|\zeta(1+m)+m(k-2)| \\
(j=2,3,4, \cdots, \mathcal{N}+2)
\end{gathered}
$$

and

$$
\begin{gathered}
\left|a_{j}\right| \leqq \frac{[\lambda+1]_{q, j-1}}{[j]_{q} !(j-1)(\mathcal{N}+1) !} \prod_{k=2}^{\mathcal{N}+3}|\zeta(1+m)+m(k-2)| \\
(j>\mathcal{N}+2) .
\end{gathered}
$$

(b) If

$$
2 m(k-1) \Re(\zeta) \leqq(k-1)^{2}(1-m)-|\zeta|^{2}(1+m),
$$

then

$$
\left|a_{j}\right| \leqq \frac{[\lambda+1]_{q, j-1}(1+m)|\zeta|}{[j]_{q} !(j-1)} \quad(j \geqq 2) .
$$

The inequalities (3.10) and (3.12) are sharp. 


\section{MAXimization of $\left|a_{3}-\mu a_{2}^{2}\right|$}

In this section, we shall need the following lemma in our discussion.

Lemma 1 ([10]). Let

$$
w(z)=\sum_{k=1}^{\infty} c_{k} z^{k} \in \Omega .
$$

If $\mu$ is any complex number, then

$$
\left|c_{2}-\mu c_{1}^{2}\right| \leqq \max \{1,|\mu|\}
$$

for any complex number $\mu$. Equality in (4.1) may be attained with the functions $w(z)=z^{2}$ and $w(z)=z$ for $|\mu|<1$ and $|\mu| \geqq 1$, respectively.

We now state and prove our main result in this section.

Theorem 3. Let the function $f(z)$ defined by (1.1) be in the normalized complexorder analytic function class $\mathcal{H}_{n, q}^{\lambda, \alpha}(\zeta, \mathcal{M})$. Suppose also that $\mu$ is any complex number. Then

$$
\left|a_{3}-\mu a_{2}^{2}\right| \leqq \frac{|\zeta(1+m)|}{2 \frac{[3]_{q} !}{[\lambda+1]_{q, 2}}\left(\frac{m+1}{m+3}\right)^{\alpha}} \max \{1,|\delta|\},
$$

where

$$
\delta=\frac{2 \frac{[3]_{q} !}{[\lambda+1]_{q, 2}}\left(\frac{m+1}{m+3}\right)^{\alpha} \mu \zeta(1+m)}{\left(\frac{[2]_{q} !}{[\lambda+1]_{q}}\right)^{2}\left(\frac{m+1}{m+2}\right)^{2 \alpha}}-[\zeta(1+m)+m] .
$$

The result is sharp.

Proof. Since $f(z) \in \mathcal{H}_{m, q}^{\lambda, \alpha}(\zeta, \mathcal{M})$, we have

$$
\begin{aligned}
w(z) & =\frac{z\left(\mathcal{N}_{m, q}^{\lambda, \alpha} f(z)\right)^{\prime}-\mathcal{N}_{m, q}^{\lambda, \alpha} f(z)}{[\zeta(1+m)-m] \mathcal{N}_{m, q}^{\lambda, \alpha} f(z)+m z\left(\mathcal{N}_{m, q}^{\lambda, \alpha} f(z)\right)^{\prime}} \\
& =\frac{\sum_{k=2}^{\infty}(k-1) \frac{[3]_{q} !}{[\lambda+1]_{q, 2}}\left(\frac{m+1}{m+3}\right)^{\alpha} a_{k} z^{k-1}}{\zeta(1+m)+\sum_{k=2}^{\infty}[\zeta(1+m)+m(k-1)] \frac{[k]_{q} !}{[\lambda+1]_{q, k-1}}\left(\frac{m+1}{m+k}\right)^{\alpha} a_{k} z^{k-1}} \\
= & \frac{\sum_{k=2}^{\infty}(k-1) \frac{[k]_{q} !}{[\lambda+1]_{q, k-1}}\left(\frac{m+1}{m+k}\right)^{\alpha} a_{k} z^{k-1}}{\zeta(1+m)}
\end{aligned}
$$




$$
\left(1+\frac{\sum_{k=2}^{\infty}[\zeta(1+m)+m(k-1)] \frac{[k]_{q} !}{[\lambda+1]_{q, k-1}}\left(\frac{m+1}{m+k}\right)^{\alpha} a_{k} z^{k-1}}{\zeta(1+m)}\right)^{-1} .
$$

We now compare the coefficients of $z$ and $z^{2}$ on both sides of the last equation (4.4). We thus obtain

$$
a_{2}=\frac{\zeta(1+m)[\lambda+1]_{q} c_{1}}{[2]_{q} !\left(\frac{m+1}{m+2}\right)^{\alpha}}
$$

and

$$
a_{3}=\frac{\zeta(1+m)}{\frac{2[3]_{q} !}{[\lambda+1]_{q, 2}}\left(\frac{m+1}{m+3}\right)^{\alpha}}\left\{c_{2}+[\zeta(1+m)+m] c_{1}^{2}\right\} \text {. }
$$

Hence

$$
a_{3}-\mu a_{2}^{2}=\frac{\zeta(1+m)}{\frac{2[3]_{q} !}{[\lambda+1]_{q, 2}}\left(\frac{m+1}{m+3}\right)^{\alpha}}\left\{c_{2}-\phi c_{1}^{2}\right\},
$$

where

$$
\phi=\frac{\frac{2[3]_{q} !}{[\lambda+1]_{q, 2}}\left(\frac{m+1}{m+3}\right)^{\alpha} \mu \zeta(1+m)}{\left(\frac{[2]_{q} !}{[\lambda+1]_{q}}\right)^{2}\left(\frac{m+1}{m+2}\right)^{2 \alpha}}-[\zeta(1+m)+m] .
$$

Taking the modulus on both sides of (4.7), we have

$$
\left|a_{3}-\mu a_{2}^{2}\right| \leqq\left|\frac{\zeta(1+m)}{\frac{2[3]_{q} !}{[\lambda+1]_{q, 2}}\left(\frac{m+1}{m+3}\right)^{\alpha}}\right| \cdot\left|c_{2}-\phi c_{1}^{2}\right| .
$$

Now, by using the above lemma in (4.9), we have

$$
\left|a_{3}-\mu a_{2}^{2}\right| \leqq\left|\frac{\zeta(1+m)}{\frac{2[3]_{q} !}{[\lambda+1]_{q, 2}}\left(\frac{m+1}{m+3}\right)^{\alpha}}\right| \max \{1,|\phi|\},
$$

where $\phi$ is given by (4.8).

Finally, the assertion (4.2) of Theorem 3 is sharp in view of the fact that the assertion (4.1) of the above lemma is known to be sharp. 


\section{CONCLUding REMARKS AND OBSERVATIONS}

In our present investigation, we have introduced and systematically studied the general class $\mathcal{H}_{m, q}^{\lambda, \alpha}(\zeta, \mathcal{M})$ of normalized analytic functions of complex order, which are connected with a $q$-analogue of integral operators. For this complex-order analytic function class, we have successfully determined a sufficient condition in terms of the coefficients and the estimates for the coefficients and a maximization theorem concerning the coefficients. Our main results are stated and proved as theorems (see Theorems 1,2 and 3). Various interesting consequences and applications of our main results are stated as corollaries.

In conclusion, it seems to worthwhile to reiterate the now well-understood fact that the results for the $q$-calculus, which we have considered in this presentation for $0<q<1$, can easily be translated into the corresponding results for the so-called $(p, q)$-calculus (with $0<q<p \leqq 1$ ) by applying some obviously trivial parametric and argument variations, the additional parameter $p$ being redundant. As a matter of fact, the so-called $(p, q)$-number $[n]_{p, q}$ is given (for $0<q<p \leqq 1$ ) by

$$
\begin{array}{rlrl}
{[n]_{(p, q)}} & := \begin{cases}\frac{p^{n}-q^{n}}{p-q} & (n \in\{1,2,3, \cdots\}) \\
0 & (n=0)\end{cases} \\
& =: p^{n-1}[n]_{\frac{q}{p}}, &
\end{array}
$$

where, for the classical $q$-number $[n]_{q}$, we have (see also Section 1 above)

$$
\begin{aligned}
{[n]_{q} } & :=\frac{1-q^{n}}{1-q} \\
& =p^{1-n}\left(\frac{p^{n}-(p q)^{n}}{p-(p q)}\right) \\
& =p^{1-n}[n]_{(p, p q)} .
\end{aligned}
$$

Furthermore, the so-called $(p, q)$-derivative or the so-called $(p, q)$-difference of a suitable function $f(z)$ is denoted by $\left(D_{p, q} f\right)(z)$ and defined, in a given subset of $\mathbb{C}$, by

$$
\left(D_{p, q} f\right)(z)= \begin{cases}\frac{f(p z)-f(q z)}{(p-q) z} & (z \in \mathbb{C} \backslash\{0\} ; 0<q<p \leqq 1) \\ f^{\prime}(0) & (z=0 ; 0<q<p \leqq 1),\end{cases}
$$

so that, clearly, we have the following connection with the familiar $q$-derivative $\left(D_{q} f\right)(z)$ used in (1.6):

$$
\left(D_{p, q} f\right)(z)=\left(D_{\frac{q}{p}} f\right)(p z) \quad \text { and } \quad\left(D_{q} f\right)(z)=\left(D_{p, p q} f\right)\left(\frac{z}{p}\right)
$$




$$
(z \in \mathbb{C} ; 0<q<p \leqq 1) .
$$

Remarkably, therefore, any claimed extensions of at least some investigations involving the classical $q$-calculus to the corresponding obviously straightforward investigations involving the $(p, q)$-calculus are somewhat inconsequential. The interested reader will find a recent investigation [25] which is intended here to provide an illustration of such transitions from the classical $q$-calculus to the $(p, q)$-calculus.

Further investigations on the applications of the $q$-calculus to meromorphic univalent and meromorphic multivalent functions along the lines of a recent work [15] may be worthy of consideration.

\section{REFERENCES}

[1] M. H. Abu-Risha, M. H. Annaby, M. E.-H. Ismail, and Z. S. Mansour, "Linear $q$-difference equations.” Z. Anal. Anwend., vol. 26, pp. 481-494, 2007.

[2] S. Agrawal and S. K. Sahoo, "A generalization of starlike functions of order $\alpha$. ." Hokkaido Math. J., vol. 46, pp. 15-27, 2017.

[3] M. K. Aouf, "Bounded p-valent Robertson functions of order $\alpha$. ." Indian J. Pure Appl. Math., vol. 16, pp. 775-790, 1985.

[4] M. K. Aouf, "Bounded spiral-like functions with fixed second coefficient." Internat. J. Math. Math. Sci., vol. 12, pp. 113-118, 1989.

[5] M. Arif, M. U. Haq, and J.-L. Liu, "A subfamily of univalent functions associated with $q$ analogue of Noor integral operator." J. Function Spaces., vol. 2018, pp. 1-5, 2018, doi: $10.1155 / 2018 / 3818915$.

[6] P. N. Chichra, "Regular functions $f(z)$ for which $z f^{\prime}(z)$ is $\alpha$-spiral-like." Proc. Amer. Math. Soc., vol. 49, pp. 151-160, 1975.

[7] M. E.-H. Ismail, E. Merkes, and D. Styer, "A generalization of starlike functions." Complex Variables Theory Appl., vol. 14, pp. 77-84, 1990, doi: 10.1080/17476939008814407.

[8] F. H. Jackson, “On $q$-functions and a certain difference operator.” Earth Environ. Sci. Trans. Royal Soc. Edinburgh., vol. 46, pp. 253-281, 1909, doi: 10.1017/S0080456800002751.

[9] F. H. Jackson, "On q-definite integrals." Quart. J. Pure Appl. Math., vol. 41, pp. 193-203, 1910.

[10] F. R. Keogh and E. P. Merkes, "A coefficient inequality for certain classes of analytic functions." Proc. Amer. Math. Soc., vol. 20, pp. 8-12, 1969, doi: 10.1090/s0002-9939-1969-0232926-9.

[11] P. K. Kulshrestha, "Bounded Robertson functions." Rend. Mat. (Ser. 6)., vol. 9, pp. 137-150, 1976.

[12] R. J. Libera, "Univalent $\alpha$-spiral functions." Canad. J. Math., vol. 19, pp. 449-456, 1967, doi: 10.4153/CJM-1967-038-0.

[13] S. Mahmood, M. Jabeen, S. N. Malik, H. M. Srivastava, R. Manzoor, and S. M. J. Riaz, "Some coefficient inequalities of $q$-starlike functions associated with conic domain defined by $q$-derivative.” J. Funct. Spaces., vol. 2018, no. Article ID 8492072, pp. 1-13, 2018, doi: 10.1155/2018/8492072.

[14] S. Mahmood, N. Raza, E. S. A. AbuJarad, G. Srivastava, H. M. Srivastava, and S. N. Malik, "Geometric properties of certain classes of analytic functions associated with a $q$-integral operator." Symmetry., vol. 11, no. Article ID 719, pp. 1-14, 2019, doi: 10.3390/sym11050719.

[15] S. Mahmood, G. Srivastava, H. M. Srivastava, E. S. A. AbuJarad, M. Arif, and F. Ghani, "Sufficiency criterion for a subfamily of meromorphic multivalent functions of reciprocal order with respect to symmetric points." Symmetry., vol. 2019, no. Article ID 764, pp. 1-7, 2019, doi: 10.3390/sym11060764. 
[16] S. Mahmood, H. M. Srivastava, N. Khan, Q. Z. Ahmad, B. Khan, and I. Ali, "Upper bound of the third Hankel determinant for a subclass of $q$-starlike functions." Symmetry., vol. 11, no. Article ID 347, pp. 1-13, 2019, doi: 10.3390/sym11030347.

[17] M. A. Nasr and M. K. Aouf, "Bounded starlike functions of complex order." Proc. Indian Acad. Sci. Math. Sci., vol. 92, pp. 97-102, 1983.

[18] L. Shi, Q. Khan, G. Srivastava, J.-L. Liu, and M. Arif, "A study of multivalent $q$-starlike functions connected with circular domain." Mathematics., vol. 7, no. Article ID 670, pp. 1-12, 2019, doi: 10.3390/math7080670.

[19] R. Singh and V. Singh, "On a class of bounded starlike functions." Indian J. Pure Appl. Math., vol. 5, pp. 733-754, 1974, doi: 10.3390/math7080670.

[20] P. I. Sižuk, "Regular functions $f(z)$ for which $z f^{\prime}(z)$ is $\theta$-spiral-shaped of order $\alpha$ (in Russian)." Sibirsk. Mat., vol. 16, pp. 1286-1290, 1975.

[21] H. M. Srivastava, Univalent functions, fractional calculus, and associated generalized hypergeometric functions. New York, Chichester, Brisbane and Toronto: In: Univalent Functions, Fractional Calculus, and Their Applications (H. M. Srivastava and S. Owa, Editors), Halsted Press (Ellis Horwood Limited, Chichester), pp. 329-354; John Wiley and Sons, 1989.

[22] H. M. Srivastava, "Operators of basic (or $q$-) calculus and fractional $q$-calculus and their applications in geometric function theory of complex analysis." Iran. J. Sci. Technol. Trans. A: Sci., vol. 44, pp. 327-344, 2020, doi: 10.1007/s40995-019-00815-0.

[23] H. M. Srivastava, Q. Z. Ahmad, N. Khan, and B. Khan, "Hankel and Toeplitz determinants for a subclass of $q$-starlike functions associated with a general conic domain." Mathematics., vol. 7, pp. 1-15, 2019, doi: 10.3390/math7020181.

[24] H. M. Srivastava, B. Khan, N. Khan, and Q. Z. Ahmad, "Coefficient inequalities for $q$-starlike functions associated with the Janowski functions." Hokkaido Math. J., vol. 48, pp. 407-425, 2019.

[25] H. M. Srivastava, N. Raza, E. S. A. AbuJarad, G. Srivastava, and M. H. AbuJarad, "FeketeSzegö inequality for classes of $(p, q)$-starlike and $(p, q)$-convex functions." Rev. Real Acad. Cienc. Exactas Fís. Natur. Ser. A Mat. (RACSAM)., vol. 113, pp. 3563-3584, 2019, doi: 10.1007/s13398019-00713-5.

[26] H. M. Srivastava, M. Tahir, B. Khan, and Q. Z. Ahmad, "Some general classes of $q$-starlike functions associated with the Janowski functions." Symmetry., vol. 11, no. Article ID 292, pp. 1-14, 2019, doi: 10.3390/sym11020292.

\section{Authors' addresses}

\section{H. M. Srivastava}

Department of Mathematics and Statistics, University of Victoria, Victoria, British Columbia V8W 3R4, Canada and Department of Medical Research, China Medical University Hospital, China Medical University, Taichung 40402, Taiwan, Republic of China and Department of Mathematics and Informatics, Azerbaijan University, 71 Jeyhun Hajibeyli Street, AZ1007 Baku, Azerbaijan

E-mail address: harimsriemath.uvic.ca

\section{Sheza M. El-Deeb}

Department of Mathematics, Faculty of Science, Damietta University, New Damietta 34517, Egypt and Department of Mathematics, College of Science and Arts in Badaya, Qassim University, Qassim, Saudi Arabia

E-mail address: shezaeldeeb@yahoo.com 